\title{
From Da Vinci Si to Da Vinci Xi: realistic times in draping and docking the robot
}

\author{
Emma M. van der Schans ${ }^{1,2}\left(\right.$ ID Marijn A. J. Hiep ${ }^{2} \cdot$ Esther C. J. Consten $^{1,3} \cdot$ Ivo A. M. J. Broeders $^{1,2}$
}

Received: 19 August 2019 / Accepted: 15 February 2020 / Published online: 20 February 2020

(c) The Author(s) 2020

\begin{abstract}
Robot-assisted surgery is assumed to be time consuming partially due to extra time needed in preparing the robot. The objective of this study was to give realistic times in Da Vinci Xi draping and docking and to analyse the learning curve in the transition from the $\mathrm{Si}$ to the $\mathrm{Xi}$ in an experienced team. This prospective study was held in a hospital with a high volume of robot-assisted surgery in general surgery, urology and gynaecology. Times from the moment patients entered the operating room until the surgeon took place behind console were precisely recorded during the first 6 weeks after the implementation of the Xi. In total, 65 procedures were performed and documented. The learning curve for the process of draping and docking the robot was reached after 21 and 18 cases, respectively. Mean times after completion of the learning curve were 5 min for draping and $7 \mathrm{~min}$ for docking and were statistically different from mean times before completion of the learning curve ( $p$ values $<0.01$ ). In dedicated teams netto extra time needed for preparing the Xi can even be reduced to just the time needed for docking. Thus, setting up the robot should have limited impact on overall time spent in the operation room.
\end{abstract}

Keywords Robot-assisted surgery $\cdot$ Docking $\cdot$ Draping $\cdot$ Learning curve $\cdot$ Time efficiency

\section{Introduction}

The use of robotic systems for surgical procedures has increased rapidly over the past 2 decades. Although robotassisted surgery has several advantages over conventional endoscopic surgery, its costs and prolonged operative times remain a subject of debate. High costs are not only ascribed to high purchase and maintenance costs of the robot, but to longer operating times as well [1-3].

Prolonged overall operating room (OR) times are partially due to extra time needed for preparing the robot (draping the robot, positioning the robot, calibrating and docking the robotic arms) [4-6]. However, focus is mainly put on total

Emma M. van der Schans

em.vander.schans@meandermc.nl

1 Department of Surgery, Meander Medical Center, Maatweg 3, Amersfoort, The Netherlands

2 Faculty of Electrical Engineering, Mathematics and Computer Science, Institute of Technical Medicine, University of Twente, Drienerlolaan 5, Enschede, The Netherlands

3 Department of Surgery, University Medical Center Groningen, Hanzeplein 1, Groningen, The Netherlands operation time and console time [7]. There is a lack of reliable data about extra time needed for setting up the robot.

Although new robotic devices are entering the surgical market, Intuitive Surgical (Sunnyvale, CA, USA) is still the market leader. In 2014, it has launched their fourth generation Da Vinci, the Da Vinci Xi Surgical System. With the recent introduction of this latest model in our experienced robotic centre, we precisely documented times of draping and docking the $\mathrm{Xi}$ with the objective to analyse the learning curve of the transition of an experienced robot OR team to a new device and to create reliable data about extra time needed to set up the Da Vinci Xi Surgical System. We hypothesized that the transition from the Si to the Xi would be mastered quickly and that extra time needed for preparing the robot can be kept to a minimum in a dedicated OR team.

\section{Methods}

\section{Study design}

This prospective study was held in a teaching hospital with a high volume of robot-assisted laparoscopic surgery. Since the purchase of a Da Vinci Si early 2011, its assistance 
has been used for a dedicated set of procedures in general surgery, urology and gynaecology. In March 2019, it was replaced by the Xi system. Set-up times of all consecutive robot-assisted surgeries performed from the start until 6 weeks after implementation of the Xi were documented by one of two independent researchers with no other obligations during the set-up phase.

\section{Surgical teams}

During this study, there were three gastro-intestinal surgeons (GI surgeons), two urologists and two gynaecologists using the robot. Thirteen well-trained scrub nurses, dedicated to robot-assisted surgery for a substantial part of their daily work, completed the teams. Although the scrub nurses rotate between the three specialties, these rotations are kept to a minimum. All team members involved had significant experience with the Si model.

Prior to definite transition of the $\mathrm{Si}$ to the $\mathrm{Xi}$, all employees of the robot OR team (consultants and nurses) attended an on-site training with the new model. The training course was held by a representative of Intuitive Surgical. This representative attended the OR during the first surgeries with the $\mathrm{Xi}$ to give instructions to the OR team if needed.

\section{Outcome measurements}

From the moment a patient entered the OR until the surgeon started at the console, time points of several different steps were recorded. All times were noted in HH:mm:ss. See Fig. 1 for the definitions of each time point and subsequent time frames differentiated. The steps 'draping robot' and 'docking robot' are seen as dedicated time needed for preparing the robotic device. Draping the robot is a step performed by two scrub nurses. It can start as soon as the patient has been positioned and the bed removed from the OR. Furthermore, draping does not interfere with the preparations done by the anaesthesiologist. This means both activities can be done in parallel. As long as the scrub nurses perform the draping efficiently and start in a timely matter, the total time spent in OR is not adversely affected.

Docking is a step performed by the surgeon, the scrub nurse, and the circulating nurse together (one for positioning and one assisting in calibrating and docking the arms).

\section{Statistical analysis}

Learning curves of robot draping and docking times were analysed using CUSUM analysis. This method enables visualization of trends within the data. It is a frequently used method to assess the process of gaining competence in performing new surgical procedures [8,9]. CUSUM is the running total of differences between the individual data points and the mean of all cases. First, procedures were ordered chronologically. The CUSUM robot draping for the first procedure was the draping time of the first case minus the mean draping time of all cases; the CUSUM robot draping for the second case was the previous case's CUSUM added to the difference between draping time of the second procedure and the mean of all cases. This was repeated for each procedure. The same analysis was done for robot docking. The learning phase was defined from the first case to the case representing

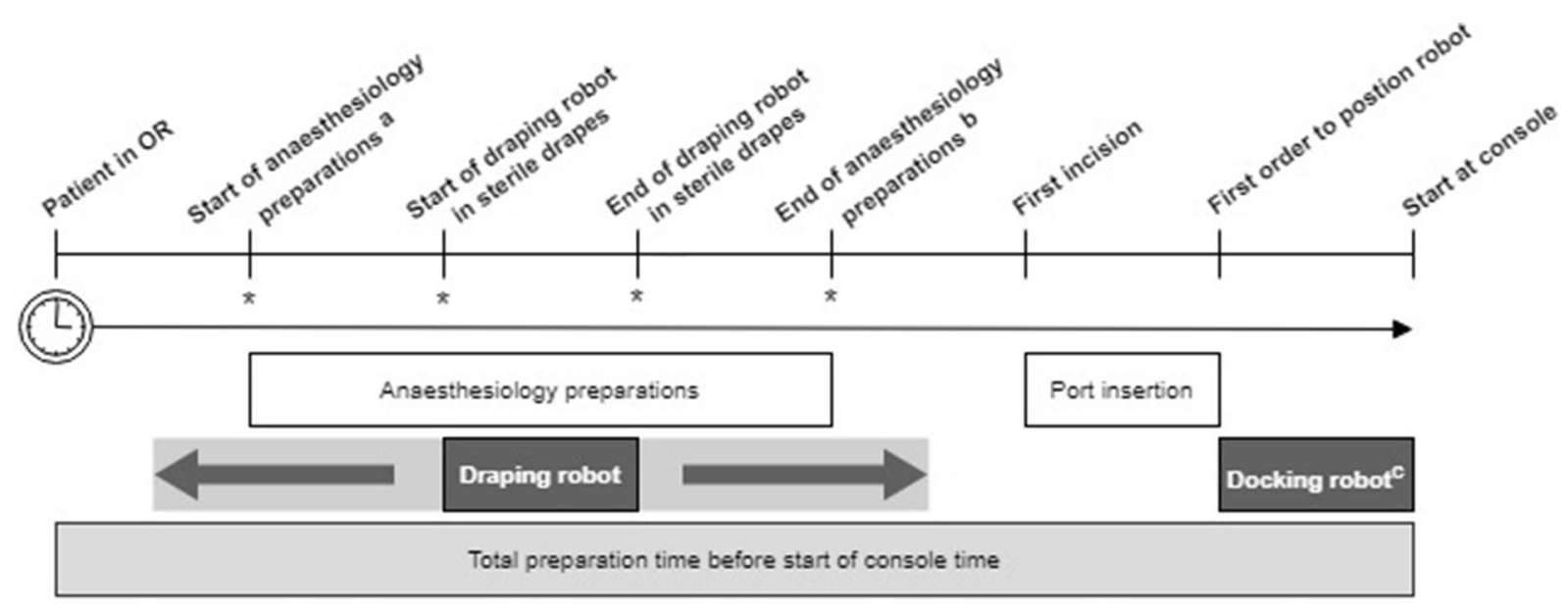

Fig. 1 Schematic time sequence illustration of OR preparations. Timeline and timeframes are not scaled. Not all steps in this schematic sequence are in chronological order necessarily. The order of the moments marked with a $*$ can shift between themselves or take place in parallel. Hence, 'Draping robot' can be executed completely or partially before, within or after 'Anaesthesiology preparations'.
This depends on OR team efficiency. a Start of pre-oxygenation can start any time after the time out has been done. b Including putting the patient in correct position for surgery but not sterile draping of the patient. c For all procedures the camera arm plus three robotic arms were connected 
the inflection point of the CUSUM curve where it changes from a positive to a negative gradient. A stable process is considered as a consistently downward trend or fluctuation around the $x$-axis [9].

Means (with standard deviation) of draping and docking times before and after this point were analysed with independent samples $t$ tests. Differences between specialties were assessed by one-way ANOVA. Statistical analysis was performed using SPSS v. 24.0 (IBM Corp., Armonk, NY, USA). A $p$ value of $<0.05$ was considered significant.

\section{Results}

All 65 robot-assisted operations performed during the first 6 weeks of using the Da Vinci Xi were recorded. The GI surgeons accounted for 26 procedures, the urologist for 25 procedures and the gynaecologists for 14 procedures. Overall mean draping and docking times were $5.6 \pm 1.4$ and $7.8 \pm 2.7 \mathrm{~min}$, respectively (Table 1 ). Times of draping and docking the robot did not statistically differ between the three surgical disciplines ( $p$ values 0.366 and 0.207 , respectively).

Times of draping and docking per consecutive procedure are plotted in Fig. 2a, b. CUSUM plots of these steps are depicted in Fig. 2c, d. The learning phase of the whole robot OR team for robot draping and docking was completed after 21 and 18 procedures, respectively. Mean time per step after completion of these learning curves was $5.0 \pm 1.2$ for draping and $6.9 \pm 1.6 \mathrm{~min}$ for docking. Mean times before and after completion of the learning curves were statistically significant ( $p$ value $\leq 0.001$; Table 1$)$.

In 36 cases (55.4\%), the draping procedure was finished before the anaesthesiology preparations were completed. In 29 cases (44.6\%), the draping procedure was still ongoing whilst the anaesthesiology preparations were finished. In all 65 cases, the duration of the anaesthesiology preparation took longer than the duration of robot draping (mean $14.6 \pm 4.6 \mathrm{~min})$. A significant difference was seen in number

Table 1 Robot draping and docking times

\begin{tabular}{lcc}
\hline Cases & Mean \pm SD (minutes) & $P$ value* \\
\hline Robot draping & & \\
$1-65$ & $5.6 \pm 1.4$ & \\
$1-21$ & $6.9 \pm 1.2$ & $<0.001$ \\
$22-65$ & $5.0 \pm 1.1$ & \\
Robot docking & & \\
$1-65$ & $7.8 \pm 2.7$ & 0.001 \\
$1-18$ & $10.3 \pm 3.3$ & \\
$19-65$ & $6.9 \pm 1.6$ & \\
\hline
\end{tabular}

*Independent samples $t$ test of cases in which the robot draping was completed before the end of anaesthesiology preparations within the three surgical disciplines $(p=0.014)$.

\section{Discussion}

Prolonged operating time remains one of the arguments against robot-assisted surgery. Extra time needed in robotassisted surgery can be split up in extra time needed for preparing the robot and in actual procedure time. Although literature holds an abundance of data about console times, there is a gap in knowledge about exact times needed in the set-up phase [5-7]. This study is the first to precisely monitor these set-up times in experienced OR teams working with a Da Vinci Xi. It is also the first to provide information on the duration of adapting to a new robotic device.

The presented data show that there is a relatively short period of 18-21 procedures for an institution with an experienced Da Vinci Si staff to adapt to the new Xi system. This transition phase leads to a limited amount of extra OR time. Draping the robot can be achieved in $5.0 \mathrm{~min}$ and can be completed within the preparation time needed by the anaesthesiologist. This means that the step of robot draping can be seen as an avoidable delay and does not necessarily affect the overall time spent in the OR. Results show, however, that this is strongly dependant on OR team efficiency. An efficient way of time management was seen in 55\% of the observed procedures in this study. A difference was found in frequency in which this most optimal form of time management happened within the OR teams of the different specialties. Attitude within a team could contribute to this.

The step of robot docking always requires extra time when comparing it to conventional laparoscopic procedures. This step can be achieved in $7 \mathrm{~min}$ on average. Unlike many other studies, docking time did not include port placement as this step is just as well performed in conventional laparoscopy and should not be considered as extra time [10,11].

Iranmanesh et al. conducted a similar study from 2006 to 2008 by precisely monitoring draping and docking times of their first experience with the Da Vinci surgical system. In their series of 96 procedures (all general surgery, eight different surgeons, unknown number of scrub nurses), they found median draping times of $22 \mathrm{~min}$ (range 9-50) [5]. This is considerably longer then the results found in our institute. Their median docking times were $10 \mathrm{~min}$ (range 2-70) which approaches our results more closely. The difference found could be due to the inexperience of their team with robotic surgery at the start of their study and the frequency of robot-assisted surgery ( 96 procedures in 30 months versus 65 procedures in 6 weeks in our study). Also, the enhancements made in the $\mathrm{Xi}$ of simpler docking, laser guided port placement and boom-mounted robotic arms could have 

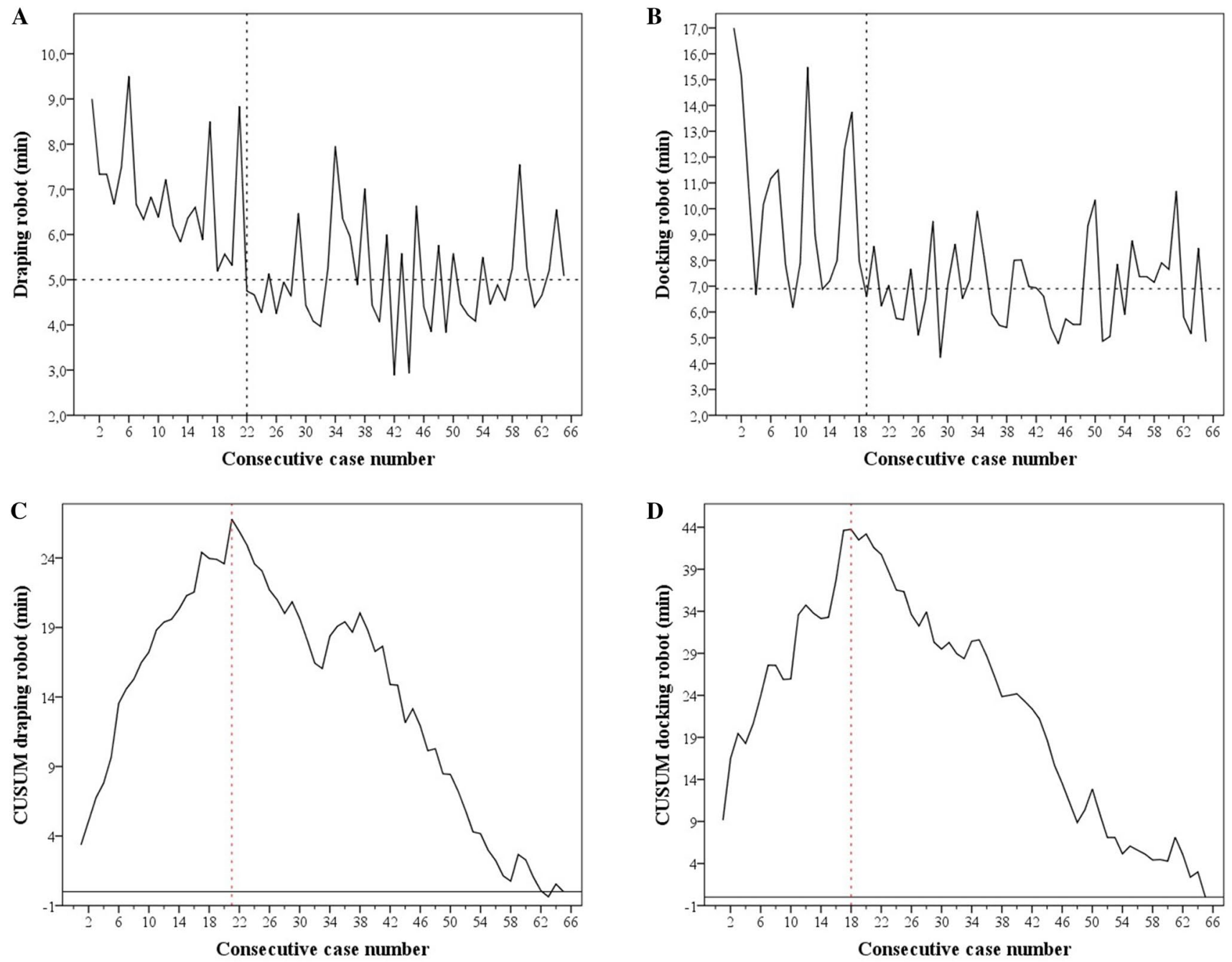

Fig. 2 Institutional robot draping and docking times and CUSUM plots. a, b Represent draping and docking times versus consecutive case number. The vertical dashed lines indicate the first case after completion of the learning phase of the associated step. The dashed

played a role in the faster times of draping and docking we found. Comparison to other studies is hard and unreliable due to unclearly described definitions of reported time frames [3, 12-16], different way of docking (three arms or single-port) $[6,17,18]$, method of data collection and/or to retrospective study designs [3, 17-19].

Robot-assisted surgery, especially the phase before the surgeon starts behind the console, is a team effort. A limitation of this study is that the composition of the OR teams per specialty was not completely fixed. This was caused by a daily rotating work schedule of our scrub nurses and more than one operating consultant per specialty. However, rotation was mainly kept within one of the surgical disciplines in an effort to minimize the variability. Due to the variable composition of the surgical teams, we were not able to analyse the learning curves per OR team separately. Instead,

horizontal lines indicate the mean time after completion of the learning phase of the associated step. $\mathbf{c}, \mathbf{d}$ Represent the plots of CUSUM versus consecutive case number. The vertical dashed lines indicate the inflection point of the CUSUM curves

an institutional learning curve was given. This represents a realistic workflow in a hospital where different specialties use the robotic device. Therefore, it enhances the generalizability of our results to other high-volume practices. Furthermore, the fact that there were no statistically significant differences found in robot draping and docking times between the three specialties supports our method.

In conclusion, both draping and docking times in robotic surgery can be kept to a minimum. CUSUM analysis showed that there is a short learning phase of 21 cases in setting up the robot when a new device is introduced. In dedicated OR teams netto extra time needed for preparing the Xi can be reduced to 7 min needed for docking. Hence, preparing the Da Vinci Xi should have a limited effect on overall time spent in the OR. With a growing number of robot-assisted surgeries, an expending availability of this technology and 
new manufacturers of surgical robots entering the market, these are valuable data for clinics implementing a (new) robot.

Acknowledgements The authors are grateful to the robot OR teams consisting of P.M. Verheijen, A.P. Lont, A. Kooistra, S.E. Schraffordt Koops, E. Lenters, P.M. Doornbos, R.M. Sonneveld, P. Christiaanse, E. van Delden, K. van Tuijl, R.G.M.J. Dorrestein, M. Postma, M.L. Langemeijer, M.J. Methorst, A. Koster, W.A.M. Verhaar, M.N.J.L. Evers, P.M.H. van den Hengel.

\section{Funding None.}

\section{Compliance with ethical standards}

Conflict of interest I.A.M.J. Broeders and E.C.J. Consten proctor for Intuitive Surgical. E.M. van der Schans and M.A.J. Hiep declare that they have no conflict of interest.

Ethical approval This article does not contain any studies with human participants or animals performed by any of the authors.

Open Access This article is licensed under a Creative Commons Attribution 4.0 International License, which permits use, sharing, adaptation, distribution and reproduction in any medium or format, as long as you give appropriate credit to the original author(s) and the source, provide a link to the Creative Commons licence, and indicate if changes were made. The images or other third party material in this article are included in the article's Creative Commons licence, unless indicated otherwise in a credit line to the material. If material is not included in the article's Creative Commons licence and your intended use is not permitted by statutory regulation or exceeds the permitted use, you will need to obtain permission directly from the copyright holder. To view a copy of this licence, visit http://creativecommons.org/licenses/by/4.0/.

\section{References}

1. Ramji KM, Cleghorn MC, Josse JM et al (2016) Comparison of clinical and economic outcomes between robotic, laparoscopic, and open rectal cancer surgery: early experience at a tertiary care center. Surg Endosc 30:1337-1343. https://doi.org/10.1007/s0046 4-015-4390-8

2. Baek S-J, Kim S-H, Cho J-S et al (2012) Robotic versus conventional laparoscopic surgery for rectal cancer: a cost analysis from a single institute in Korea. World J Surg 36:2722-2729. https:// doi.org/10.1007/s00268-012-1728-4

3. Morelli L, Di Franco G, Lorenzoni V et al (2019) Structured cost analysis of robotic TME resection for rectal cancer: a comparison between the da Vinci $\mathrm{Si}$ and $\mathrm{Xi}$ in a single surgeon's experience. Surg Endosc 33:1858-1869. https://doi.org/10.1007/s0046 4-018-6465-9

4. Park EJ, Baik SH (2016) Robotic surgery for colon and rectal cancer. Curr Oncol Rep 18:5. https://doi.org/10.1007/s1191 2-015-0491-8

5. Iranmanesh P, Morel P, Wagner OJ et al (2010) Set-up and docking of the da Vinci ${ }^{\circledR}$ surgical system: prospective analysis of initial experience. Int J Med Robot Comput Assist Surg 6:57-60. https ://doi.org/10.1002/rcs.288
6. Iranmanesh P, Morel P, Buchs NC et al (2013) Docking of the da Vinci Si surgical system ${ }^{\circledR}$ with single-site technology. Int J Med Robot Comput Assist Surg 9:12-16. https://doi.org/10.1002/ res. 1481

7. Fung AK-Y, Aly EH (2013) Robotic colonic surgery. Dis Colon Rectum 56:786-796. https://doi.org/10.1097/DCR.0b013e3182 $85 \mathrm{~b} 810$

8. Bokhari MB, Patel CB, Ramos-Valadez DI et al (2011) Learning curve for robotic-assisted laparoscopic colorectal surgery. Surg Endosc 25:855-860. https://doi.org/10.1007/s00464-010-1281-x

9. Cundy TP, Gattas NE, White AD, Najmaldin AS (2015) Learning curve evaluation using cumulative summation analysis-a clinical example of pediatric robot-assisted laparoscopic pyeloplasty. J Pediatr Surg 50:1368-1373. https://doi.org/10.1016/j.jpeds urg.2014.12.025

10. Souders CP, Catchpole K, Hannemann A et al (2019) Flow disruptions in robotic-assisted abdominal sacrocolpopexy: does robotic surgery introduce unforeseen challenges for gynecologic surgeons? Int Urogynecol J. https://doi.org/10.1007/s00192-01903929-6

11. Alenizi AM, Valdivieso R, Rajih E et al (2015) Factors predicting prolonged operative time for individual surgical steps of robotassisted radical prostatectomy (RARP): a single surgeon's experience. Can Urol Assoc J 9:E417-E422. https://doi.org/10.5489/ cuaj. 2805

12. Alfieri S, Butturini G, Boggi U et al (2019) Short-term and longterm outcomes after robot-assisted versus laparoscopic distal pancreatectomy for pancreatic neuroendocrine tumors (pNETs): a multicenter comparative study. Langenbeck's Arch Surg 404:459468. https://doi.org/10.1007/s00423-019-01786-x

13. Hagen ME, Jung MK, Ris F et al (2017) Early clinical experience with the da Vinci Xi Surgical System in general surgery. J Robot Surg 11:347-353. https://doi.org/10.1007/s11701-016-0662-0

14. Tamhankar AS, Jatal S, Saklani A (2016) Total robotic radical rectal resection with da Vinci Xi system: single docking, single phase technique. Int J Med Robot 12:642-647. https://doi.org/10.1002/ rcs. 1734

15. Abdel Raheem A, Sheikh A, Kim DK et al (2017) Da Vinci Xi and $\mathrm{Si}$ platforms have equivalent perioperative outcomes during robot-assisted partial nephrectomy: preliminary experience. J Robot Surg 11:53-61. https://doi.org/10.1007/s11701-016-0612-x

16. Ngu JC-Y, Sim S, Yusof S et al (2017) Insight into the da Vinci ${ }^{\circledR}$ $\mathrm{Xi}$ - technical notes for single-docking left-sided colorectal procedures. Int J Med Robot 13:e1798. https://doi.org/10.1002/rcs.1798

17. Choi SH, Hong S, Kim M et al (2019) Robotic-assisted laparoscopic myomectomy: the feasibility in single-site system. Obstet Gynecol Sci 62:56-64. https://doi.org/10.5468/ogs.2019.62.1.56

18. Raimondi P, Marchegiani F, Cieri M et al (2018) Is right colectomy a complete learning procedure for a robotic surgical program? J Robot Surg 12:147-155. https://doi.org/10.1007/s1170 1-017-0711-3

19. Yim GW, Eoh KJ, Chung YS et al (2018) Perioperative outcomes of 3-arm versus 4-arm robotic radical hysterectomy in patients with cervical cancer. J Minim Invasive Gynecol 25:823-831. https ://doi.org/10.1016/j.jmig.2017.12.009

Publisher's Note Springer Nature remains neutral with regard to jurisdictional claims in published maps and institutional affiliations. 